\title{
Optical Metrology in the Transportation Industry
}

\author{
Frank Chen \\ Ford Motor Co. \\ 20000 Rotunda Drive \\ Dearborn, Michigan 48121 \\ E-mail: fchen1@ford.com
}

\author{
Lianxiang Yang \\ Oakland University \\ Department of Mechanical Engineering \\ Lab. of Optical Measurement and Quality Inspection \\ Rochester, Michigan 48309 \\ E-mail: yang2@oakland.edu
}

Optical metrology has experienced an escalation in research, development, and applications within the transportation industry. With advances in digital imaging devices, evolution in computer technologies, including algorithms and software, and development of novel loading and stressing schemes, it has become an indispensable technique that enables the transportation industry to develop, design, and manufacture products with highly desired features, useful functions, better quality, and lower cost in less time.

This special section serves as a forum to share the latest advances of optical measurement methods and their novel applications in the industry, including automotive, aeronautic/astronautic, and other related fields. It includes a variety of development and application examples, such as: detection of spheres in a 3-D point cloud using a Hough transform, stabilization of a fiber optical sensor with feedback loop technology, demonstration of the advantages of fiber optical sensors that can be used in the long-term monitoring and gathering of information from reinforced concrete bridges, insightful results on mixed mode crack propagation, and the pros and cons of shearography versus thermography on nondestructive testing in composite materials and structures.

There are total of 15 papers in this section. Ogundana et al. developed a novel calibration method using a Hough transform that extends the detection of circles in 2-D images to spheres in 3-D point clouds. It becomes practical to use by reducing the excessive memory storage requirement with a sparse 3-D matrix model scheme. This makes 3-D spherical type object measurement more accurate and faster.

The second group contains papers on digital image correlation techniques. Sutton et al. provide an excellent application example using the 3-D digital image correlation method to measure a 3-D displacement field, both dynamic and static, over a large displacement range. It results in some insightful conclusions on mixed mode crack propagations. Siebert et al. give a comprehensive error source analysis on the ever-increasingly popular digital image correlation method, which can be used to measure large range displacement and strain both in static mode and dynamic phase. The errors include both calibration and data reconstruction sources. Huang and Waas describe a digital speckle method using the fast Fourier transform (FFT) in the frequency domain to determine the displacement, by overlapping the fraction of subelement sizes, to accurately determine the FFT peak to increase accuracy and sensitivity. Yang and Wu devised a digital image correlation measurement tool, with a double long focus microscope, which can be used to measure strain less than $1 \mu \varepsilon$.

The third group deals with thermography and shearography. Hung et al. provide a comprehensive review on two often used composite debonding or delimination detection methods: shearography and pulsed thermography. The paper discusses the strengths and limitations, and most importantly, the complementary aspects of the two methods. Shepard et al. developed an automatic defect detection and identification procedure using thermography that can be applied in the automotive industry. Bhaduri, Mohan, and Kothiyal deal with digital speckle pattern interferometry and digital shearography using the spatial phase shift method, which has the advantage over the temporal method of less sensitivity to environmental disturbance. Nondestructive examples are given as well.

The fourth group concerns fiber optical sensors. Watkins, Fonda, and Nanni provide a comparison between fiber optical interferometric sensors, strain gauges, and analytical methods on the measurement of strain of a reinforced concrete bridge that was repaired. It shows that the fiber optical sensor is consistent with the analytical method, no failure or degradation, as opposed to strain gauges. Xie, Lu, and Zhang developed a stabilization technique on a fiber optic interferometer using an electric feedback loop that can truly take advantage of its high 
resolution and sensitivity, which is more suitable for inprocess, precise measurement.

The last group includes a variety of method developments and applications. Liu and Yang developed a regional phase unwrapping method based on fringe estimation and phase map segmentation. Greene, Yates, and Patterson developed a method to detect a crack in a rail foot using an infrared emission method. Pena et al. measure the velocity of a projectile that impacts a spacecraft or aircraft using a microcontrolled optoelectronic system. This method provides reasonable accuracy, but has some advantages over other methods, such as simplicity, costeffectiveness, robustness, and a large measurement range. $\mathrm{Ji}$ et al. employ the isodyne photoelasticity method to measure stress in directional Plexiglas. Using the frequency domain phase shift method, the phase map of the fringes is obtained. Kuang, Hong, and Feng describe a single method that can simultaneously measure twodimensional rotational angles with a collimation laser.

We would like to thank all of the authors for their high-quality contributions and all of the reviewers for their valuable comments and effort to ensure the high quality of the publication. We also would like to thank the Editor, Dr. O'Shea, the Managing Editor, Ms. Labes, the Peer Review Coordinator, Ms. Munger, the Editorial Assistant, Ms. McCullough, and the other SPIE staff for providing both the opportunity and the technical support for a special section on this subject. We believe this special section will further promote research and applications of optical metrology in the transportation industry.

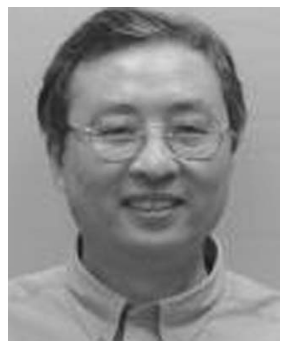

Frank Chen received his PhD degree in mechanical system engineering from Oakland University. He is currently a CBP technical leader at Ford Motor Company and an adjunct professor at Wayne State University. He has diversified experience in applying optical metrology in vehicle noise, vibration, and harshness reduction, component stress optimization, and flow visualization, which includes developed laser metrology based methodology in measuring brake pad damping and in nondestructive inspecting brake pad insulator debonding. He served as a co-guest editor on the special sections on "Optical Metrology: Development and Application in the Transportation Industry" for Optical Engineering in 1998,2000 , and 2003. He is also presently an editorial board member of the Journal of Holography and Speckle. His laser metrology work has been published in four books and has appeared in more than 45 technical articles including seven invited papers. $\mathrm{He}$ holds nine patents and has received several awards including a significant achievement award, an innovation award, and a global customer quality award from Ford Motor Company.

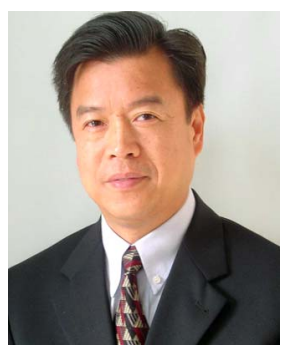

Lianxiang Yang received his $\mathrm{PhD}$ in mechanical engineering from the University of Kassel, Germany. He has been an associate professor in the Department of Mechanical Engineering at Oakland University in Rochester, Michigan, USA, since 2001. Prior to joining Oakland University, he was a R\&D scientist at JDS-Uniphase, Canada, from 2000 to 2001, a senior engineer at Dantec-Ettemeyer AG, Germany, from 1998 to 2000, a research and senior research fellow at the University of Kassel, Germany, from 1991 to 1998, and a lecturer and associate professor at Hefei University of Technology, China, from 1986 to 1991. Dr. Yang's research interests and competencies lie in the areas of advance optical measuring techniques and their applications in automotive, aeronautic/ astronautic, and biomedical engineering, with a focus on digital, quantitative, and nondestructive measurement and inspection of 3-D contour, 3-D deformations, strain/stress, vibration, and characterization of advanced materials and biomaterials. He has authored and co-authored about 100 scientific research papers, two books, and one book chapter, and has eight patents in these areas. Dr. Yang is also presently an associate technical editor of Material Evaluation and an editorial board member of the Journal of Holography and Speckle. 